\title{
Perfil psicológico de atletas paraolímpicos brasileiros
}

\author{
Dietmar Samulski ${ }^{1}$ e Franco Noce ${ }^{2}$
}

\section{RESUMO}

O objetivo deste estudo é apresentar e discutir os resultados da preparação psicológica realizada com 64 atletas de oito diferentes esportes. A avaliação foi realizada nos Centros de Treinamento Paraolímpicos no Rio de Janeiro, São Paulo e Recife. Foram aplicados testes de personalidade, testes de motivação, questionário de estresse e testes psicométricos (percepção, concentração e tempo de reação). Foi detectada diferença significativa em alguns parâmetros psicológicos ao comparar os atletas de acordo com o sexo e os tipos de deficiência (física, visual ou mental). De forma geral, o motivo mais importante para iniciar uma atividade esportiva foi o prazer da prática e a necessidade de reabilitação. Os principais motivos para praticar esportes foram a competição e o desejo de superar limites. A maioria dos atletas mencionou os seguintes fatores estressantes: problemas de sono, pressão de vencer e conflitos interpessoais. A maioria dos atletas apresentou bons resultados na percepção e no tempo de reação. Baseados nesses resultados foi desenvolvida uma orientação individual no período de preparação para os Jogos Paraolímpicos.

Palavras-chave: Avaliação psicológica. Testes de personalidade. Testes psicométricos.

\section{ABSTRACT}

\section{Psychological profile of Brazilian paralympic athletes}

The purpose of this study was to present and discuss the results of a psychological evaluation carried out with 64 athletes from eight different sports disciplines. The evaluation was carried out in the Paralympic training centers in

1. Doutor em Psicologia do Esporte pela Universidade de Colônia, Alemanha.

2. Mestre em Treinamento Desportivo/Psicologia do Esporte pela Universidade Federal de Minas Gerais - UFMG.

Endereço para correspondência:

Rua dos Construtores, 464

30830-550 - Belo Horizonte, MG

E-mail: fnoce2000@yahoo.com.br sam@eef.ufmg.br
Rio de Janeiro, São Paulo, and Recife, applying personality tests, motivation tests, stress inventory, and psychometric tests (perception, concentration and reaction time). Significant differences were detected in some psychological parameters comparing male and female athletes and athletes with different kinds of disability (physical, visual or mental). In general, the most important motives to begin a sports activity were pleasure and the need for rehabilitation. The main motive for actual sports practice was competition and the desire to overcome limits. The majority of the athletes mentioned the following stressing factors: sleeping problems, pressure to win, and interpersonal conflicts. Most of the athletes showed good results in perception and reaction time. Based on these results individual guidance was developed to prepare the athletes for the Paralympic Games.

Key words: Psychological evaluation. Personality testing. Psychometric testing.

\section{INTRODUÇÃO}

O desporto paraolímpico tem conquistado um espaço cada vez maior no cenário nacional e mundial. Dessa forma, tem-se evidenciado a necessidade de melhoria das condições de treinamento e também de melhor suporte para o atleta paraolímpico dedicar-se às atividades esportivas. Nesse contexto, a preparação psicológica aparece como mais uma ferramenta de auxílio ao atleta ${ }^{1}$.

Nessa oportunidade, a avaliação psicológica foi solicitada pelo Comitê Paraolímpico Brasileiro (CPB) com a finalidade de obter informações básicas sobre o perfil psicológico de cada atleta (65 atletas) e sobre sua situação sociocultural. Os resultados das avaliações (psicológicas, médicas, fisiológicas e biomecânicas) serviram como base científica para subsidiar e orientar melhor os trabalhos dos técnicos na fase de preparação dos atletas para as Paraolimpíadas em Sydney 2000.

Além disso, os dados levantados servirão como referência para as próximas avaliações e preparações de atletas. Dessa forma, é possível observar e analisar o desenvolvi- 
mento psicossocial e o da performance de cada atleta a longo prazo (nos próximos quatro-oito anos) e elaborar parâmetros para comparações em nível internacional (instalar um banco de dados sobre os atletas brasileiros no CPB).

Os objetivos gerais da preparação psicológica nesse período foram obter informações sobre o perfil psicológico de cada atleta e do grupo como um todo; dar suporte científico para o trabalho dos técnicos na área da psicologia do esporte; e fundamentar cientificamente o trabalho da preparação psicológica dos atletas para Sydney. Os objetivos específicos foram detectar problemas psicológicos específicos relacionados com a competição esportiva; analisar os objetivos, metas e motivos para a prática esportiva de cada atleta; identificar fatores estressantes e motivadores antes e durante a competição; e analisar o tempo de reação, o nível de duração e a velocidade da percepção dos atletas.

\section{Fundamentação teórica}

A avaliação de atletas paraolímpicos, no aspecto psicológico, é um procedimento complexo em função da heterogeneidade do grupo, de seu perfil e da influência de inúmeras variáveis presentes no contexto. Capacidades e habilidades básicas avaliadas em um primeiro momento, tais como a motivação, o estresse, o tempo de reação e a velocidade de percepção, são essenciais para a definição de um plano de trabalho eficiente em qualquer modalidade.

\section{Estresse}

De forma geral, "o estresse é produto da interação do homem com o seu meio ambiente físico e sociocultural"2. De acordo com Nitsch ${ }^{3}$, existem fatores pessoais (processos psíquicos e somáticos) e fatores ambientais (ambiente físico e social) que se interagem no processo de surgimento e gerenciamento do estresse.

A concepção de estresse, compartilhada entre diferentes autores ${ }^{4-6}$ mostra concordância unânime no que se refere à associação do estresse com estado de desestabilização psicofísica ou a pertubação do equilíbrio pessoa-meio ambiente. O conceito de estresse como reação, segundo Selye ${ }^{6}$, compreende a "totalidade das reações de adaptação orgânica, as quais objetivam a manutenção ou reestabelecimento do equilíbrio interno e/ou externo".

O conceito de estresse, de acordo com Nitsch ${ }^{3}$, pode ser compreendido como um produto tridimensional (figura 1). Os conceitos biológicos, psicológicos e sociológicos devem ser sempre pensados em dependência recíproca, pois processos psíquicos e sociais são ligados, de determinada forma, a processos biológicos. Processos sociais, por sua vez, são influenciados através de aspectos psicológicos e ambos podem tornar-se grandes influenciadores de respostas biológicas.

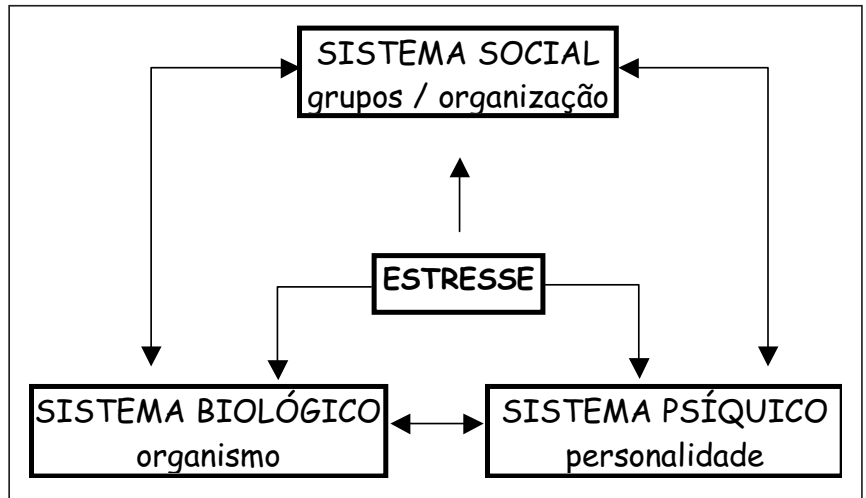

Fig. 1 - Estresse como um produto tridimensional (Nitsch, 1981:53)

\section{Motivação}

Segundo Samulski ${ }^{7}$, "a motivação é caracterizada como um processo ativo, intencional e dirigido a uma meta, o qual depende da interação de fatores pessoais (intrínsecos) e ambientais (extrínsecos)". Segundo esse modelo (figura 2), a motivação apresenta determinante energética (nível de ativação) e uma determinante de direção do comportamento (intenções, interesses, motivos e metas). Baseandose nesse conceito de motivação podem-se distinguir técnicas de ativação (activation-control) e técnicas de estabelecer metas (goal-setting strategies).

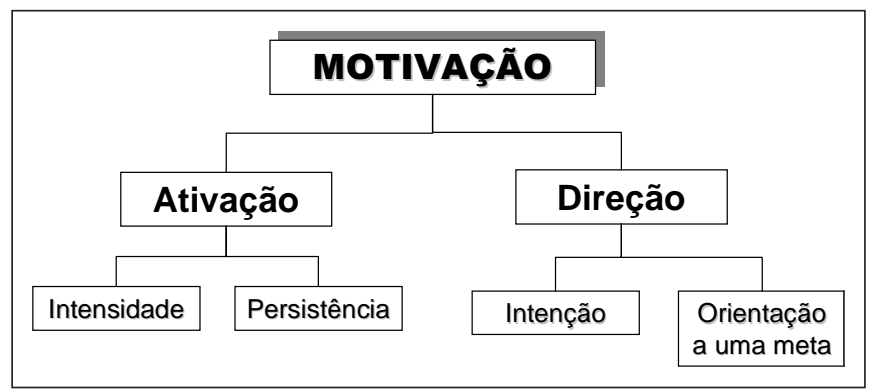

Fig. 2 - Determinantes da motivação (Samulski, 1995:55)

Existem, de acordo com Samulski ${ }^{8}$, várias teorias de motivação que podem influenciar a prática da atividade física e esportivas dos atletas paraolímpicos brasileiros. Dentre elas, podem-se destacar duas:

- A motivação para a prática esportiva que, de acordo com Weinberg e Gould ${ }^{9}$, envolve a interação de fatores pessoais e ambientais, sendo que a importância desses fatores pode mudar, dependendo das necessidades e oportunidades atuais.

- A teoria da motivação do rendimento: que explica a motivação para o rendimento como o resultado da interação de fatores pessoais e situacionais, em que os autores ${ }^{10,11}$ destacam cinco componentes fundamentais (fatores da per- 
sonalidade e motivos, fatores situacionais, tendências resultantes, reações emocionais e comportamento de rendimento).

\section{MÉTODOS}

Participaram da avaliação 64 atletas de nove modalidades. Em relação ao sexo do grupo, a maioria é do masculino $(82,8 \%)$. Já em relação ao tipo de deficiência, nota-se que $61 \%$ foram do tipo físico-motor, $23,4 \%$ mental e $15,6 \%$ visual.

A pesquisa foi solicitada pelo Comitê Paraolímpico Brasileiro, onde, em reunião, foram definidos os critérios para sua realização. Todos os atletas foram informados sobre os procedimentos da pesquisa, sendo esta de caráter anônimo.

Os instrumentos utilizados na coleta de dados foram a ficha de dados individuais e também dois testes do aparelho Multipsy-82 $1^{12}$.

\section{A. Ficha de dados individuais}

\section{A.1. Perfil geral do atleta}

- dados demográficos;

- iniciação esportiva;

- incentivo à prática;

- objetivos e metas pessoais;

\section{A.2. Questionário de motivação para prática esportiva}

- motivos iniciais para a prática esportiva

- motivos atuais para a prática esportiva

- motivos de abandono da prática esportiva

\section{A.3. Teste de estresse psíquico}

- avaliação de 35 condições e fatores

\section{B. Testes no aparelho Multipsy-821}

\section{B.1. Teste de tempo de reação simples}

- duas tentativas com a mão de preferência

\section{B.2. Teste de velocidade de percepção}

- uma tentativa

\section{Teste de reação simples}

Este módulo representa, simplesmente, a velocidade em que um indivíduo é capaz de responder a um estímulo quando este é apresentado ${ }^{13}$. Isso é importante para atividades que requerem reação rápida a um estímulo inesperado.

$\mathrm{Na}$ linguagem coloquial, o termo "tempo de reação" é entendido como a média do tempo entre um estímulo e algum tipo de reação observável pelo indivíduo. O uso científico desse termo, dessa maneira, coincide com a defini- ção científica proposta, de acordo com a qual "tempo de reação" é o intervalo entre a aplicação do estímulo e o início da resposta ${ }^{14}$.

\section{Teste de velocidade de reação}

Os dados foram coletados nas cidades de Recife e São Paulo. Foram utilizadas duas salas, sendo uma para realizar as entrevistas e outra para os testes no aparelho Multip$s y-821$. As entrevistas eram realizadas pelos avaliadores com perguntas padronizadas, porém de forma a adaptar a linguagem ao nível de compreensão de cada atleta. Durante os testes no aparelho Multipsy-821, os atletas recebiam informações padronizadas e o teste era demonstrado de forma a facilitar a assimilação das informações.

A análise dos dados foi descritiva, composta por procedimentos de média, desvio padrão e distribuição de frequiência. Para comparação dos grupos sexo e tipo de deficiência foi utilizada a análise de variância, seguida do teste de comparações múltiplas de Scheffé. Os procedimentos foram realizados no pacote estatístico SPSS for Windows.

\section{RESULTADOS}

\section{Perfil do grupo}

Antes de iniciarmos a análise dos dados coletados, é importante ter um perfil do grupo avaliado nesses dois períodos de coleta de dados (Recife e São Paulo). Pode-se verificar (tabela 1) que foram avaliados 64 atletas de nove modalidades, sendo que o maior grupo é o da natação, com 17 atletas $(26,6 \%)$. A determinação do perfil do grupo avaliado é importante para a definição dos procedimentos a serem utilizados no período preparatório e competitivo ${ }^{15}$.

Outras informações são igualmente importantes para caracterizar o perfil do grupo. Verifica-se que o grupo apresentou idade média de 26,19 anos $( \pm 5,49)$ e que iniciou a prática esportiva em média aos 12 anos $( \pm 5,59)$, porém

\section{TABELA 1}

Distribuição dos atletas por modalidade

\begin{tabular}{lrrr}
\hline Valid & N & \multicolumn{1}{c}{$\%$} & \% acumulado \\
& & & \\
Basquete DM & 12 & 18,8 & 18,8 \\
Futebol de PC & 11 & 17,2 & 35,9 \\
Halterofilisno & 3 & 4,7 & 40,6 \\
Ciclismo & 2 & 3,1 & 43,8 \\
Judô & 5 & 7,8 & 51,6 \\
Atletismo - Corridas & 6 & 9,4 & 60,9 \\
Natação & 17 & 26,6 & 87,5 \\
Atletismo - Lançamentos & 4 & 6,3 & 93,8 \\
Tênis de mesa & 4 & 6,3 & 100,0 \\
Total & 64 & 100,0 & \\
\hline
\end{tabular}


começou a competir apenas aos 17 anos $( \pm 5,16)$. Quem mais motivou esses atletas a iniciarem sua prática esportiva, de forma geral, foram os pais $(32,8 \%)$ e os amigos $(26,6 \%)$.

\section{TABELA 2}

Objetivos e metas no esporte (geral)

\begin{tabular}{lrrrr}
\hline Valid & N & $\%$ & $\begin{array}{c}\% \\
\text { válido }\end{array}$ & $\begin{array}{c}\% \\
\text { acumulado }\end{array}$ \\
& & & & 6,7 \\
Participar nas Olimpíadas & 10 & 5,2 & 6,7 & 27,5 \\
Ser campeão & 31 & 16,1 & 20,8 & 40,3 \\
Conquistar medalhas & 19 & 9,9 & $\mathbf{1 2 , 8}$ & 4,3 \\
Vencer/ficar bem colocado & 6 & 3,1 & 4,0 & 44,3 \\
Superar limites & 15 & 7,8 & 10,1 & 54,4 \\
Ser reconhecido & 18 & 9,4 & 12,1 & 66,4 \\
Conseguir patrocínio & 4 & 2,1 & 2,7 & 69,1 \\
Retorno financeiro & 11 & 5,7 & 7,4 & 76,5 \\
Socialização & 8 & 4,2 & 5,4 & 81,9 \\
Melhorar desempenho & 14 & 7,3 & 9,4 & 91,3 \\
Qualidade de vida/saúde & 6 & 3,1 & 4,0 & 95,3 \\
Viajar & 3 & 1,6 & 2,0 & 97,3 \\
Outros & 4 & 2,1 & 2,7 & 100,0 \\
Total & 149 & 77,6 & 100,0 & \\
Missing & & & & \\
Não respondeu & & & & \\
Total & 43 & 22,4 & & \\
\hline
\end{tabular}

Atualmente, verificamos que o volume de treinamento, de forma geral, é em média executado quatro vezes por semana, com duração média de duas horas e meia por sessão de treinamento ${ }^{12}$.

\section{Objetivos e metas no esporte}

Para analisar os objetivos e metas pessoais no esporte (tabela 2), foi utilizada uma questão aberta com até três possibilidades de resposta, sendo que nem sempre o atleta indicava três objetivos. Dessa forma, após a categorização das mesmas, observamos 12 grupos principais.

Como falado anteriormente, que nem todos os atletas responderam três objetivos, eliminamos as respostas em branco $(22,4 \%)$ e utilizamos o percentual válido para as análises. Dessa forma, nota-se que os três principais objetivos do grupo, de forma geral, foram: "ser campeão" $(20,8 \%)$; "conquistar medalhas" $(12,8 \%)$ e "ser reconhecido" $(12,1 \%)$.

Ao compararmos os objetivos de acordo com o tipo de deficiência (tabela 3), nota-se um quadro bem interessante. O objetivo "ser campeão" não foi o principal objetivo para os deficientes visuais. Na opinião destes, "superar limites" e "retorno financeiro" são as principais metas no esporte.

\section{Motivação para a prática esportiva}

Nesta etapa foram analisados os diferentes motivos que levaram os atletas a iniciarem a prática esportiva, bem como

TABELA 3

Objetivos e metas no esporte (deficiência)

\begin{tabular}{|c|c|c|c|c|c|c|}
\hline & \multicolumn{6}{|c|}{ Deficiência } \\
\hline & \multirow{2}{*}{\multicolumn{2}{|c|}{$\begin{array}{c}\text { Mental } \\
\text { Objetivos } \\
\text { no esporte }\end{array}$}} & \multirow{2}{*}{\multicolumn{2}{|c|}{$\begin{array}{c}\text { Visual } \\
\text { Objetivos } \\
\text { no esporte }\end{array}$}} & \multirow{2}{*}{\multicolumn{2}{|c|}{$\begin{array}{c}\text { Físico-motora } \\
\text { Objetivos } \\
\text { no esporte }\end{array}$}} \\
\hline & & & & & & \\
\hline & $\mathbf{N}$ & $\%$ & $\mathbf{N}$ & $\%$ & $\mathbf{N}$ & $\%$ \\
\hline Participar nas Olimpíadas & & & 2 & 7,1 & 8 & 8,7 \\
\hline Ser campeão & 8 & 27,6 & 2 & 7,1 & 21 & 22,8 \\
\hline Conquistar medalhas & 4 & 13,8 & 4 & 14,3 & 11 & 12,0 \\
\hline Vencer/ficar bem colocado & 1 & 3,4 & 2 & 7,1 & 3 & 3,3 \\
\hline Superar limites & 2 & 6,9 & 5 & 17,9 & 8 & 8,7 \\
\hline Ser reconhecido & 3 & 10,3 & 4 & 14,3 & 11 & 12,0 \\
\hline Conseguir patrocínio & 1 & 3,4 & 1 & 3,6 & 2 & 2,2 \\
\hline Retorno financeiro & 3 & 10,3 & 5 & 17,9 & 3 & 3,3 \\
\hline Socialização & 3 & 10,3 & & & 5 & 5,4 \\
\hline Melhorar desempenho & 2 & 6,9 & 1 & 3,6 & 11 & 12,0 \\
\hline Qualidade de vida/saúde & & & 1 & 3,6 & 5 & 5,4 \\
\hline Viajar & 2 & 6,9 & & & 1 & 1,1 \\
\hline Outros & & & 1 & 3,6 & 3 & 3,3 \\
\hline Total & 29 & 100,0 & 28 & 100,0 & 92 & 100,0 \\
\hline
\end{tabular}


os motivos que os mantêm praticando esportes e os possíveis motivos que os levariam a abandonar a prática.

\section{Motivos de manutenção da prática}

Neste caso foram apresentados 16 motivos (categorizados de outros estudos) em uma tabela na qual os atletas deveriam avaliar a importância de cada um em uma escala de quatro valores, variando entre 0 (motivo sem importância) e 3 (motivo decisivo).

De forma geral, verificou-se que os principais motivos que mantêm os atletas praticando esportes são: o "prazer da prática" $(2,75 \pm 0,47)$; "gostar de competir" $(2,70 \pm$ $0,55)$; e "fazer amizades" $(2,57 \pm 0,59)$. Em contrapartida, os motivos menos importantes foram o "retorno financeiro" $(1,67 \pm 1,11)$ e o "status social" $(1,81 \pm 0,81)$. O elevado desvio padrão do motivo retorno financeiro confirma a observação feita durante as entrevistas de que, apesar de os atletas ou da maioria destes não receber remuneração pela prática, estes têm o desejo de um dia ser reconhecidos e receber remuneração satisfatória de forma a possibilitar maior dedicação à modalidade.

A tabela 4 pode confirmar essa informação, em que $28,6 \%$ consideram o retorno financeiro como um fator decisivo.

Em relação aos grupos de acordo com o tipo de deficiência (tabela 5), verificou-se diferença significativa em cinco motivos. Os DMs foram significativamente mais motivados que os DFs em relação aos motivos "aumentar co- nhecimentos no esporte" ( $\mathrm{p}<0,01)$, "aprender a cooperar" $(p<0,05)$ e "para viajar" ( $p<0,01)$. Os DMs, juntamente com os DVs, foram mais motivados que os DFs em "fazer amizades" ( $p<0,001)$. Finalmente, os DVs consideram mais importante que os DFs ( $\mathrm{p}<0,05)$. Pode-se verificar, ainda, que os motivos considerados menos importantes foram praticamente os mesmos para todos os grupos, porém, com níveis de importância diferentes.

\section{Motivos de abandono}

A avaliação destes motivos foi realizada da mesma forma que os motivos de manutenção. Assim, o atleta deveria indicar, em um rol de 10 motivos, o nível de importância de cada um que o levaria a abandonar a atividade esportiva.

De forma geral (tabela 6), verifica-se que os principais motivos que poderiam levar os atletas a abandonar o esporte são os "problemas com a saúde/lesões" $(1,73 \pm 1,23)$ e também a "falta de prazer" $(1,63 \pm 1,22)$. A tabela 6 mostra mais detalhadamente esses resultados, em que o que menos importaria aos atletas é a "falta de talento", juntamente com a "falta de contatos sociais".

Em relação à análise dos motivos que influenciam no abandono da prática em relação ao tipo de deficiência, observou-se também que não foram encontradas diferenças significativas. Verificou-se que os motivos de "falta de prazer" e "problemas de saúde/lesões" são comuns a todos os grupos.

TABELA 4

Freqüência dos motivos que mantêm o grupo praticando esportes (geral)

\begin{tabular}{|c|c|c|c|c|c|c|c|c|}
\hline & \multicolumn{2}{|c|}{$\begin{array}{c}\text { Sem } \\
\text { importância }\end{array}$} & \multicolumn{2}{|c|}{$\begin{array}{c}\text { Pouca } \\
\text { importância }\end{array}$} & \multicolumn{2}{|c|}{ Importante } & \multicolumn{2}{|c|}{ Decisivo } \\
\hline & $\mathbf{N}$ & $\%$ & $\mathbf{N}$ & $\%$ & $\mathbf{N}$ & $\%$ & $\mathbf{N}$ & $\%$ \\
\hline 1. Prazer da prática & & & 1 & 1,6 & 14 & 22,2 & 48 & 76,2 \\
\hline 2. Sentir-se realizado & 1 & 1,6 & 2 & 3,2 & 23 & 37,1 & 36 & 58,1 \\
\hline 3. Aprender novos movimentos & 3 & 4,8 & 9 & 14,5 & 23 & 37,1 & 27 & 43,5 \\
\hline 4. Aumentar conhec. no esporte & 2 & 3,2 & 9 & 14,3 & 20 & 31,7 & 32 & 50,8 \\
\hline 5. Melhorar desempenho esportivo & & & & & 30 & 46,9 & 34 & 53,1 \\
\hline 6. Gostar de competir & & & 3 & 4,7 & 13 & 20,3 & 48 & 75,0 \\
\hline 7. Por ter sucesso no esporte & 1 & 1,6 & 3 & 4,7 & 20 & 31,3 & 40 & 62,5 \\
\hline 8. Conhecer limites & 3 & 4,8 & 3 & 4,8 & 15 & 24,2 & 41 & 66,1 \\
\hline 9. Pelo retorno financeiro & 13 & 20,6 & 13 & 20,6 & 19 & 30,2 & 18 & 28,6 \\
\hline 10. Gostar de desafios & & & 6 & 9,5 & 24 & 38,1 & 33 & 52,4 \\
\hline 11. Fazer amizades & & & 3 & 4,8 & 21 & 33,3 & 39 & 61,9 \\
\hline 12. Pelo status social & 3 & 4,8 & 18 & 29,0 & 29 & 46,8 & 12 & 19,4 \\
\hline 13. Ser reconhecido & 3 & 4,8 & 10 & 15,9 & 25 & 39,7 & 25 & 39,7 \\
\hline 14. Aprender a cooperar & & & 5 & 8,2 & 31 & 50,8 & 25 & 41,0 \\
\hline 15. Incentivo da família e amigos & 1 & 1,6 & 4 & 6,3 & 20 & 31,3 & 39 & 60,9 \\
\hline 16. Para viajar & 1 & 1,6 & 6 & 9,4 & 22 & 34,4 & 35 & 54,7 \\
\hline
\end{tabular}


TABELA 5

Motivos que mantêm o grupo praticando esportes (deficiência)

\begin{tabular}{|c|c|c|c|c|c|c|}
\hline & \multicolumn{6}{|c|}{ Deficiência } \\
\hline & \multicolumn{2}{|c|}{ Mental } & \multicolumn{2}{|c|}{ Visual } & \multicolumn{2}{|c|}{ Físico-motora } \\
\hline & Média & D. padrão & Média & D. padrão & Média & D. padrão \\
\hline 1. Prazer da prática & 2,71 & 0,61 & 2,90 & 0,32 & 2,72 & 0,46 \\
\hline 2. Sentir-se realizado & 2,77 & 0,44 & 2,60 & 0,52 & 2,41 & 0,72 \\
\hline 3. Aprender novos movimentos* & 2,50 & 0,65 & 2,60 & 0,70 & 1,97 & 0,91 \\
\hline 4. Aumentar conhecimentos no esporte ${ }^{*}$ & 2,71 & 0,47 & 2,70 & 0,67 & 2,05 & 0,89 \\
\hline 5. Melhorar desempenho esportivo & 2,60 & 0,51 & 2,50 & 0,53 & 2,51 & 0,51 \\
\hline 6. Gostar de competir & 2,93 & 0,26 & 2,70 & 0,48 & 2,62 & 0,63 \\
\hline 7. Por ter sucesso no esporte & 2,33 & 0,90 & 2,60 & 0,52 & 2,62 & 0,59 \\
\hline 8. Conhecer limites & 2,57 & 0,76 & 2,80 & 0,42 & 2,42 & 0,89 \\
\hline 9. Pelo retorno financeiro & 1,60 & 1,18 & 1,67 & 1,22 & 1,69 & 1,08 \\
\hline 10. Gostar de desafios & 2,43 & 0,65 & 2,50 & 0,71 & 2,41 & 0,68 \\
\hline 11. Fazer amizades*** & 2,93 & 0,27 & 2,90 & 0,32 & 2,36 & 0,63 \\
\hline 12. Pelo status social & 2,14 & 0,95 & 1,80 & 0,79 & 1,68 & 0,74 \\
\hline 13. Ser reconhecido & 2,36 & 1,01 & 2,20 & 1,03 & 2,05 & 0,76 \\
\hline 14. Aprender a cooperar* & 2,69 & 0,48 & 2,40 & 0,70 & 2,18 & 0,61 \\
\hline 15. Incentivo da família e amigos & 2,73 & 0,46 & 2,50 & 0,53 & 2,44 & 0,79 \\
\hline 16. Para viajar** & 2,80 & 0,41 & 2,70 & 0,67 & 2,21 & 0,77 \\
\hline
\end{tabular}

TABELA 6

Freqüência dos motivos que podem levar ao abandono do esporte (geral)

\begin{tabular}{|c|c|c|c|c|c|c|c|c|}
\hline & \multicolumn{2}{|c|}{$\begin{array}{c}\text { Sem } \\
\text { importância }\end{array}$} & \multicolumn{2}{|c|}{$\begin{array}{c}\text { Pouca } \\
\text { importância }\end{array}$} & \multicolumn{2}{|c|}{ Importante } & \multicolumn{2}{|c|}{ Decisivo } \\
\hline & $\mathbf{N}$ & $\%$ & $\mathbf{N}$ & $\%$ & $\mathbf{N}$ & $\%$ & $\mathbf{N}$ & $\%$ \\
\hline 1. Falta de talento & 32 & 51,6 & 8 & 12,9 & 16 & 25,8 & 6 & 9,7 \\
\hline 2. Pressão de vencer & 25 & 40,3 & 14 & 22,6 & 13 & 21,0 & 10 & 16,1 \\
\hline 3. Monotonia ou carga excessiva nos treinos & 23 & 36,5 & 19 & 30,2 & 14 & 22,2 & 7 & 11,1 \\
\hline 4. Falta de prazer & 17 & 27,0 & 11 & 17,5 & 13 & 20,6 & 22 & 34,9 \\
\hline 5. Falta de tempo $\mathrm{p} /$ outras atividades & 16 & 26,2 & 20 & 32,8 & 19 & 31,1 & 6 & 9,8 \\
\hline 6. Falta de sucesso & 26 & 41,9 & 10 & 16,1 & 18 & 29,0 & 8 & 12,9 \\
\hline 7. Falta de contatos sociais & 28 & 47,5 & 17 & 28,8 & 10 & 16,9 & 4 & 6,8 \\
\hline 8. Problemas com saúde/lesões & 16 & 25,4 & 10 & 15,9 & 12 & 19,0 & 25 & 39,7 \\
\hline 9. Conflitos com técnicos/companheiros & 23 & 37,1 & 18 & 29,0 & 13 & 21,0 & 8 & 12,9 \\
\hline 10. Conflitos com familiares & 27 & 43,5 & 17 & 27,4 & 9 & 14,5 & 9 & 14,5 \\
\hline
\end{tabular}

\section{Estresse psíquico}

O teste é composto por 35 situações que podem exercer influência positiva ou negativa no rendimento do atleta ${ }^{12}$. $\mathrm{O}$ atleta deveria avaliar cada situação utilizando uma escala likert de sete fatores, sendo:

+3: influência muito positiva

+2 : influência positiva

+1: influência pouco positiva

0: nenhuma influência

-1: influência pouco negativa
-2 : influência pouco negativa

-3 : influência pouco negativa

A figura 3 apresenta o resultado médio do grupo nas 35 situações. Observa-se que a grande maioria das situações foi considerada estressante para o grupo como um todo; o fator mais estressante foi "dormir mal na noite anterior à competição".

Observa-se também que, embora muitas situações sejam aparentemente neutras, o desvio padrão relativamente alto indica que existem opiniões diversas. Assim, uma aná- 
lise mais detalhada, comparando o grupo de acordo com o tipo de deficiência, poderá apresentar perfis diferenciados em relação às diferentes situações propostas no teste.

Ao analisar o teste de estresse psíquico em relação aos tipos de deficiência (tabela 7), verifica-se que cada grupo tem um perfil bem definido. Os fatores que mais estressam, por exemplo, para os DMs foi "errar no fim da competição" $(-0,79 \pm 1,58)$, porém o desvio padrão é muito elevado, o que nos leva a crer que existe um conflito em relação a essa situação.

Já para os DVs, a situação mais estressante é "não ter condições adequadas de treino" $(-2,70 \pm 0,67)$. É impor-

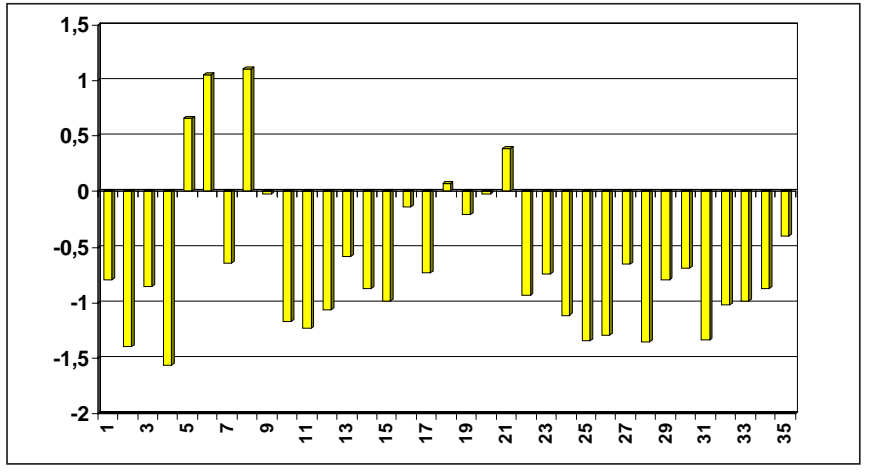

Fig. 3 - Análise geral do teste de estresse psíquico

TABELA 7

Teste de estresse psíquico (deficiência)

\begin{tabular}{|c|c|c|c|c|c|c|}
\hline & \multicolumn{6}{|c|}{ Deficiência } \\
\hline & \multicolumn{2}{|c|}{ Mental } & \multicolumn{2}{|c|}{ Visual } & \multicolumn{2}{|c|}{ Físico-motora } \\
\hline & Média & D. padrão & Média & D. padrão & Média & D. padrão \\
\hline 1. Erros no início** & 0,46 & 1,76 & $-1,30$ & 0,95 & $-1,08$ & 1,53 \\
\hline 2. Erros no fim da competição & $-0,79$ & 1,58 & $-1,80$ & 1,14 & $-1,50$ & 1,39 \\
\hline 3. Demora para iniciar a competição** & 0,21 & 1,31 & $-1,60$ & 1,35 & $-1,05$ & 1,41 \\
\hline 4. Dormir mal na noite anterior à competição* & $-0,69$ & 1,44 & $-2,30$ & 1,16 & $-1,67$ & 1,40 \\
\hline 5. Ser o favorito & 1,08 & 1,71 & 0,20 & 1,48 & 0,63 & 1,95 \\
\hline 6. O adversário é o favorito & 0,85 & 2,03 & 1,90 & 1,60 & 0,89 & 1,80 \\
\hline 7. Pressão de outros para vencer & $-0,25$ & 1,60 & $-1,00$ & 1,89 & $-0,67$ & 1,72 \\
\hline 8. Cobrança de si mesmo para vencer & 1,08 & 1,80 & 1,00 & 2,40 & 1,13 & 1,89 \\
\hline 9. Maus resultados em competições anteriores & $-0,08$ & 1,98 & $-0,30$ & 1,42 & 0,08 & 1,65 \\
\hline 10. Condicionamento físico inadequado & $-0,21$ & 1,85 & $-1,20$ & 1,87 & $-1,51$ & 1,55 \\
\hline 11. Preparação técnica inadequada** & $-0,08$ & 1,98 & $-2,20$ & 0,79 & $-1,36$ & 1,46 \\
\hline 12. Falta de preparação psicológica** & 0,00 & 1,41 & $-2,20$ & 1,03 & $-1,15$ & 1,57 \\
\hline 13. Conflitos com treinador & 0,23 & 2,05 & $-0,30$ & 1,16 & $-0,92$ & 1,72 \\
\hline 14. Conflitos com companheiros & $-0,69$ & 1,55 & $-0,60$ & 0,70 & $-1,00$ & 1,36 \\
\hline 15. Conflitos com familiares & $-0,38$ & 1,50 & $-1,00$ & 1,15 & $-1,18$ & 1,23 \\
\hline 16. Bom rendimento inesperado adversário* & 0,93 & 1,44 & $-0,70$ & 1,25 & $-0,38$ & 1,68 \\
\hline 17. Maus rendimentos nos treinos* & 0,07 & 1,69 & $-1,70$ & 1,34 & $-0,77$ & 1,78 \\
\hline 18. Críticas do treinador na competição & 0,58 & 1,83 & 0,30 & 1,95 & $-0,16$ & 1,88 \\
\hline 19. Críticas dos companheiros na competição & 0,43 & 1,74 & $-0,20$ & 1,99 & $-0,45$ & 1,48 \\
\hline 20. Ter perdido para o mesmo adversário & 0,46 & 1,56 & 0,00 & 2,24 & $-0,18$ & 1,76 \\
\hline 21. Comportamento da torcida contra & $-0,43$ & 1,74 & 0,60 & 1,26 & 0,63 & 1,75 \\
\hline 22. Nervosismo excessivo & $-0,38$ & 1,71 & $-0,90$ & 1,60 & $-1,13$ & 1,58 \\
\hline 23. Sentir dor durante os treinos* & 0,21 & 1,97 & $-1,40$ & 1,17 & $-0,92$ & 1,28 \\
\hline 24. Sentir dor antes ou durante a competição* & $-0,15$ & 1,86 & $-1,10$ & 1,10 & $-1,44$ & 1,25 \\
\hline 25. Alimentação inadequada** & $-0,38$ & 1,71 & $-2,60$ & 0,52 & $-1,33$ & 1,66 \\
\hline 26. Condições inadequadas de treino** & $-0,43$ & 1,83 & $-2,70$ & 0,67 & $-1,24$ & 1,48 \\
\hline 27. Aparelhos ortopédicos inadequados & $-0,21$ & 1,19 & 0,00 & 0,00 & $-0,94$ & 1,33 \\
\hline 28. Materiais e equip. esportivos inadequados & $-0,54$ & 1,51 & $-1,78$ & 1,39 & $-1,53$ & 1,35 \\
\hline 29. Falta de reconhecimento esportivo** & 0,14 & 1,41 & $-1,56$ & 1,13 & $-0,95$ & 1,41 \\
\hline 30. Falta de apoio familiar*** & 0,92 & 1,80 & $-0,90$ & 1,29 & $-1,18$ & 1,55 \\
\hline 31. Falta de patrocínio** & $-0,17$ & 2,04 & $-2,10$ & 0,88 & $-1,50$ & 1,31 \\
\hline 32. Falta de ass. fisiot. e médica fora da seleção** & 0,29 & 2,23 & $-1,90$ & 1,37 & $-1,26$ & 1,43 \\
\hline 33. Falta de ass. psicológica fora da seleção** & 0,15 & 1,72 & $-1,70$ & 1,34 & $-1,18$ & 1,41 \\
\hline 34. Probl. transporte para treino e competição** & 0,29 & 1,73 & $-1,50$ & 1,18 & $-1,13$ & 1,34 \\
\hline 35. Problemas de alojamento*** & 1,21 & 1,72 & $-1,20$ & 1,23 & $-0,77$ & 1,46 \\
\hline
\end{tabular}

${ }^{*} \mathrm{p}<0,05 ;{ }^{*} \mathrm{p}<0,01 ; * * * \mathrm{p}<0,001$ 
tante citar que os DVs são o grupo que atribui, em média, maior nível de estresse às situações. Os DFs já consideram "dormir mal na noite anterior à competição" $(-1,67 \pm 1,40)$ como o fator mais estressante.

Em relação aos fatores motivantes, também é possível observar diferenças entre os grupos. Um situação estranha é os DMs considerarem "problemas de alojamento" (1,21 \pm 1,72 ) como a situação mais motivadora, porém o desvio padrão elevado sugere conflitos neste item. Os DVs consideram o fato de o "adversário ser o favorito" $(1,90 \pm 1,60)$ mais motivante. Finalmente, os DFs já acham mais positiva "a cobrança de si mesmo para vencer" $(1,13 \pm 1,89)$. Verifica-se que outros estudos aplicados a outras modalidades apresentaram resultados semelhantes em algumas das variáveis observadas ${ }^{16-18}$.

\section{Testes Multipsy}

A avaliação foi composta por dois testes que tinham como objetivo mensurar algumas capacidades psicológicas específicas.

- Tempo de reação simples

Visa mensurar o tempo de reação através de um estímulo visual simples.

- Tachistoscopy

Visa mensurar a velocidade e qualidade de percepção.

Os testes, em ambas as cidades, foram realizados em uma sala em condições padrão (temperatura e nível de ruído ambiente) favoráveis.

\section{Teste de reação simples}

O teste consiste no indivíduo responder a um estímulo ótico no menor tempo possível. Foram realizadas duas tentativas com os atletas, sendo utilizada em ambas a mão de preferência. Para efeito de cálculo a primeira tentativa foi desprezada, eliminando o efeito de aprendizagem. Os resultados foram expressos em milissegundos, sendo apresentados ainda o desvio padrão, o coeficiente de variação e a média dos resultados do grupo.

O resultado geral (tabela 8) mostrou que o grupo apresenta velocidade de reação satisfatória $(229,41 \mathrm{~ms})$; foi ve-

\section{TABELA 8}

Resultado geral do teste de reação simples

\begin{tabular}{lrrrr}
\hline & Mín. & Máx. & Média & $\begin{array}{l}\text { Desvio } \\
\text { padrão }\end{array}$ \\
Tempo de reação 1 & 195 & 525 & 255,91 & 69,19 \\
Desvio padrão 1 & 16 & 224 & 75,45 & \\
Tempo de reação 2 & 169 & 441 & 229,41 & 52,12 \\
Desvio padrão 2 & 11 & 193 & 52,73 & \\
\hline
\end{tabular}

rificada melhora significativa $(\mathrm{p}<0,001)$ no desempenho entre a primeira e a segunda tentativa (através do teste $t$ para amostras pareadas). Além da resposta mais rápida, observou-se que a estabilidade das respostas também foi uma realidade, em que o desvio padrão saiu de $75,45 \mathrm{~ms}$ (na $1^{\text {a }}$ tentativa) para $52,73 \mathrm{~ms}$ (na 2 a tentativa). Pode-se observar na tabela 8 que os resultados foram relativamente heterogêneos.

Os resultados por tipo de deficiência (figura 4) mostram que o grupo DFs possui o melhor tempo de reação quando comparado com os outros grupos $(\mathrm{p}<0,05)$. Destaca-se ainda que os grupos também melhoraram na $2^{2}$ tentativa.

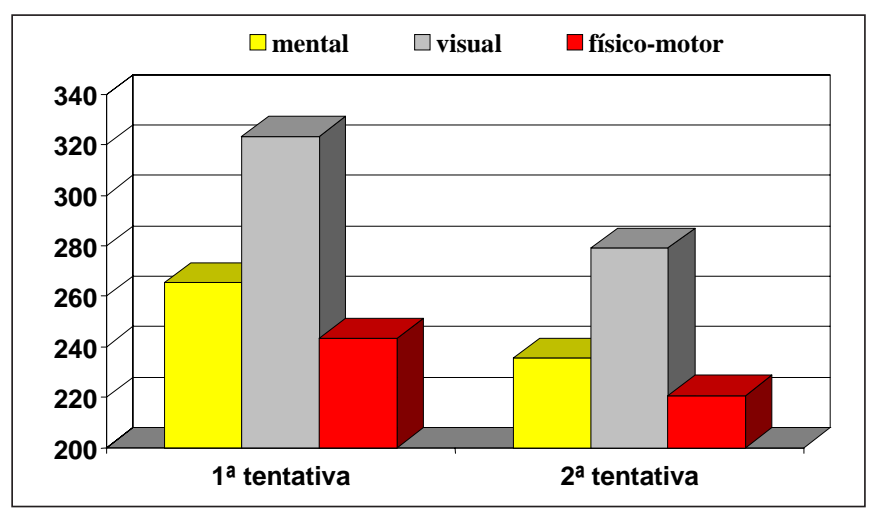

Fig. 4 - Tempo de reação simples (deficiência)

É importante citar que alguns DVs realizaram o teste de percepção tátil-cinestésica através do toque do operador. Este método foi bastante interessante, uma vez que alguns desses atletas têm que reagir aos sinais enviados pelo guia (como no caso das corridas). A tabela 9 mostra mais detalhadamente os resultados obtidos pelos grupos.

Outros estudos demonstraram que o resultado do tempo de reação pode ser influenciado por uma série de variáveis. Samulski et al. ${ }^{19}$ verificaram que a privação de sono associada a esforço físico intenso pode causar alterações na velocidade de reação. Já Paula Filho ${ }^{20}$ cita que em um esforço de $85 \%$ do $\mathrm{VO}_{2}$ max, mesmo ocorrendo a fadiga física, o tempo de reação não ficará comprometido e a ativação do sistema nervoso central (SNC) estará aumentada. Foi verificado ainda que o nível de concentração em uma tarefa de longa duração combinado com outras variáveis pode afetar significativamente o desempenho do tempo de reação ${ }^{21}$.

\section{Taquistoscopia}

O teste consiste no indivíduo identificar uma série de estímulos (números de três dígitos). Os estímulos são apresentados, inicialmente, de forma bem rápida e pouco níti- 
TABELA 9

Análise descritiva dos tempos de reação em função do tipo de deficiência

\begin{tabular}{|c|c|c|c|c|c|c|c|}
\hline & & & $\mathbf{N}$ & Média & D. padrão & Mín. & Máx. \\
\hline \multirow{4}{*}{$\begin{array}{l}\text { Tempo de } \\
\text { reação - } \\
\text { 1a tentativa }\end{array}$} & Deficiência & Mental & 15 & 265,27 & 60,59 & 195 & 414 \\
\hline & & Visual & 5 & 323,20 & 121,19 & 203 & 475 \\
\hline & & Físico-motora & 38 & 243,37 & 59,91 & 195 & 525 \\
\hline & & Total & 58 & 255,91 & 69,19 & 195 & 525 \\
\hline \multirow{4}{*}{$\begin{array}{l}\text { Tempo de } \\
\text { reação - } \\
\text { 2a tentativa }\end{array}$} & Deficiência & Mental & 15 & 235,47 & 51,13 & 179 & 346 \\
\hline & & Visual & 5 & 279,20 & 108,84 & 183 & 441 \\
\hline & & Físico-motora & 39 & 220,69 & 38,94 & 169 & 385 \\
\hline & & Total & 59 & 229,41 & 52,12 & 169 & 441 \\
\hline
\end{tabular}

da (esta forma de apresentação pode ser identificada nos resultados como tempo 1). A medida que o indivíduo não consegue responder adequadamente aos estímulos, estes vão-se tornando cada vez mais nítidos e o tempo de permanência no visor vai aumentando gradativamente. Este teste visa mensurar uma série de capacidades psicológicas, entre elas: percepção, atenção e memória a curto pra$\mathrm{ZO}^{13}$.

Pode-se verificar (tabela 10) que, de forma geral, o grupo foi relativamente heterogêneo neste teste. O melhor resultado é o indivíduo responder a apenas cinco estímulos, sendo todos corretos e no tempo 1 (o mais rápido e menos nítido).

Já em relação aos tipos de deficiência (tabela 11), não foi possível nenhum DV realizar este teste. Assim, comparando os DMs com os DFs, verificou-se que existe uma dife-

\section{TABELA 10}

Resultado do teste de velocidade de percepção (geral)

\begin{tabular}{lrrrrc}
\hline & N & Mín. & Máx. & Média & D. padrão \\
& & & & & \\
№ de estímulos & 53 & 5 & 17 & 6,32 & 2,22 \\
№ corretos & 53 & 3 & 8 & 4,79 & 0,93 \\
Tempo & 53 & 1 & 13 & 2,25 & 2,43 \\
\hline
\end{tabular}

rença significativa nos valores de números de estímulos ( $\mathrm{p}$ $<0,05)$ e tempo ( $\mathrm{p}<0,05)$. Isso significa que os DFs conseguem perceber e memorizar estímulos que aparecem mais rapidamente e menos nítidos.

O estudo de Samulski et al. ${ }^{19}$ demonstrou que a privação de sono associada a intensa atividade física com pressão psicológica pode afetar em até $25 \%$ o rendimento do indivíduo na variável velocidade de percepção.

\section{CONCLUSÕES E CONSEQÜÊNCIAS}

Baseado nos resultados das avaliações psicológicas e com o apoio da literatura, torna-se possível o desenvolvimento de um programa de treinamento psicológico para os atletas $^{1}$. A intervenção in loco do psicólogo do esporte auxilia a observação e determinação de metodologias mais apropriadas ao contexto do desporto paraolímpico.

Durante as intervenções in loco, o psicólogo responsável conversou com os atletas e técnicos sobre problemas existentes e aplicou algumas técnicas psicológicas como: de concentração e motivação, de relaxamento, de visualização e de controle emocional ${ }^{22}$. Com os times de basquetebol e futebol foram aplicadas também técnicas de dinâmica de grupo com a finalidade de desenvolver o espírito de time e união de grupo.

TABELA 11

Resultado do teste de velocidade de percepção (deficiência)

\begin{tabular}{|c|c|c|c|c|c|c|c|c|}
\hline & \multicolumn{8}{|c|}{ Deficiência } \\
\hline & \multicolumn{4}{|c|}{ Mental } & \multicolumn{4}{|c|}{ Físico-motora } \\
\hline & Média & D. padrão & Mín. & Máx. & Média & D. padrão & Mín. & Máx. \\
\hline № de estímulos & 7,71 & 3,27 & 5 & 17 & 5,84 & 1,46 & 5 & 11 \\
\hline № corretos & 4,71 & 1,49 & 3 & 8 & 4,84 & 0,64 & 3 & 6 \\
\hline Tempo & 3,79 & 3,85 & 1 & 13 & 1,71 & 1,35 & 1 & 7 \\
\hline
\end{tabular}




\section{RECOMENDAÇÕES}

Baseadas nas experiências adquiridas durante o trabalho com os atletas paraolímpicos brasileiros e na convivência com os técnicos, apresentam-se abaixo algumas recomendações para ações futuras na área do esporte paraolímpico:

1) Criar uma comissão interdisciplinar permanente de avaliação e de acompanhamento científico com o objetivo de dar continuidade a um trabalho interdisciplinar que mostrará grande eficiência.

2) Ampliar a oferta de testes psicológicos com o fim de obter mais informações, especialmente na área sociocultural.

3) Oferecer cursos de capacitação para os técnicos na área de Psicologia do Esporte com o fim de melhorar o entendimento destes sobre os testes psicológicos e também que adquiram técnicas de motivação e comunicação.

4) Formar núcleos de treinamento psicológico em diferentes regiões do Brasil, considerando as necessidades e peculiaridades de cada região. $\mathrm{O}$ trabalho de treinamento será avaliado e supervisionado pelo psicólogo responsável do СРB.

5) Incentivar a pesquisa na área da Psicologia do Esporte aplicada a pessoas portadoras de deficiência e divulgar os resultados através dos meios de comunicação.

\section{AGRADECIMENTOS}

- Comitê Paraolímpico Brasileiro (CPB)

- Secretaria Nacional de Esportes

- Rede Cenesp/Unifesp

- Rede Cenesp/UfmG

- Associação Fundo de Incentivo à Psicofarmacologia (Afip)

- Universidade Federal de Minas Gerais

\section{REFERÊNCIAS}

1. Samulski D. Psicologia do esporte: um manual para a educação física, fisioterapia e psicologia. São Paulo: Manole, 2002.

2. Samulski D, Noce F, Chagas M. Estresse. In: Samulski D, editor. Psicologia do esporte: um manual para a educação física, fisioterapia e psicologia. São Paulo: Manole, 2002.

3. Nitsch JR. Stress: theorien, untersuchungen und massnahmen. Bern/Stuttgart/Wien: Verlag Hans Huber, 1981.

4. McGrath J. Stress und verhalten in organizationen. In: Nitsch JR, editor. Stress: theorien, untersuchungen und massnahmen. Bern/Stuttgart/Wien: Verlag Hans Huber, 1981.
5. Samulski D, Chagas MH, Nitsch J. Stress: teorias básicas. Belo Horizonte: Costa \& Cupertino, 1996.

6. Selye H. Geschichte und Grundzüge des Stresskonzepts. In: Nitsch JR, editor. Stress: theorien, untersuchungen und massnahmen. Bern/Stuttgart/ Wien, Verlag Hans Huber, 1981.

7. Samulski D. Psicologia do esporte: teoria e aplicação prática. Belo Horizonte: Imprensa UFMG, 1995.

8. Samulski D. Motivação. In: Samulski D, editor. Psicologia do esporte: um manual para a educação física, fisioterapia e psicologia. São Paulo: Manole, 2002.

9. Weinberg RS, Gould D. Foundations of sport and exercise psychology. Champaign: Human Kinetics, 1999.

10. Atkinson JW. The main stream of achievement oriented activity. In: Atkinson JW, Raynor J, editors. Motivation and achievement. New York: Halstead, 1974:13-41.

11. McClelland D. The achievement society. New York: Free Press, 1961.

12. Samulski D, Noce F. Avaliação psicológica do esporte. In: Mello MT, organizador. Preparação física para atletas paraolímpicos. São Paulo: Atheneu, 2002.

13. Biodata. Multipsy 821: User manual. Frankfurt: Biodata, 1988.

14. Pilz LH. Beschreibung und Analyse von inadäquaten und richtigen Reaktionen. Unveröff. Diss., Leopold-Franzens-Universität, Innsbruck, 1982.

15. Samulski D, Noce F, et al. Análise dos dados psicossociais. In: Resultado da avaliação dos jogos da juventude 1997. Brasília: Publicações Indesp, 1998:11-24.

16. Chagas MH. Análise do estresse psíquico na competição em jogadores de futebol de campo das categorias juvenil e júnior. Belo Horizonte: Escola de Educação Física da UFMG, Dissertação de mestrado em Ciências do Esporte, 1995.

17. Noce F. Análise do estresse psíquico em atletas de voleibol de alto nível: um estudo comparativo entre gêneros. Belo Horizonte: Escola de Educação Física da UFMG, Dissertação de mestrado em Educação Física/ Treinamento Esportivo, 1999.

18. Samulski D, Noce F. Diagnóstico e controle do estresse no esporte. In: Samulski D, editor. Novos conceitos em treinamento esportivo. Série Indesp de publicações em Ciências do Esporte. Indesp: Brasília, 1999.

19. Samulski D, Noce F, Dias JC. Respostas psicológicas em situação de privação de sono. Temas Atuais em Educação Física em Esportes IV. Editora Health: Belo Horizonte, 1999.

20. Paula Filho U. Efeito do exercício aeróbico de alta intensidade até a exaustão sobre o tempo de reação auditiva, o tempo de reação visual e a fadiga mental avaliada através da frequiência de vibração e fusão. Belo Horizonte: Escola de Educação Física, Dissertação de mestrado em Ciências do Esporte, 1993.

21. Samulski DM, Noce F, Araújo MA, Castro MM. Análise da fadiga mental e dos tempos de reação em motoristas de ônibus In: II Seminário de Comportamento Motor, 2000, São Paulo. Anais do II Seminário de Comportamento Motor, São Paulo: Imprensa Universitária USP, 2000:1:2543.

22. Becker Junior B, Samulski D. Manual de treinamento psicológico para o esporte. Rio Grande do Sul: Edelbra, 1998. 